only $33 \%$ of infants were scanned between 2 and 7 days, the optimum time for parents and caregivers to make decisions to limit treatment. In the Hammersmith hospital, the decision to withdraw intensive care was based on the history, clinical state, cranial ultrasound findings, EEG data, as well as lesions seen on the MRI scan. Brainstem injury had a similar predictive value for death in infants who died early, after withdrawal of intensive care, or later.

\title{
PREDICTIVE VALUE OF EARLY A-EEG IN HIE OUTCOME
}

Researchers at Wayne State University and other US Pediatric Centers examined the predictive value of the early amplitude integrated electroencephalogram (aEEG) and stage of encephalopathy among infants with hypoxic-ischemic encephalopathy (HIE) eligible for whole-body hypothermia. Neonates were enrolled prospectively if moderate or severe HIE occurred at $<6$ hours, and a 30-minute aEEG was obtained at $<9$ hours of age. The primary outcome was death or moderate/severe disability at 18 months. aEEGs were categorized as normal $(n=12)$, discontinuous normal voltage $(n=12)$, or abnormal, with burst suppression $(n=22)$, continuous low voltage $(n=26)$, or flat tracing $(n=36)$. At 18-month follow-up, 53 infants $(49 \%)$ had died or were disabled. Severe HIE and an abnormal aEEG were related to the primary outcome with univariate analysis, whereas severe HIE alone was predictive of outcome with multivariate analysis. Based on the Sarnat staging criteria at $<6$ hours of age, of a total of 108 infants enrolled, $71(66 \%)$ had moderate HIE and 37 (34\%) severe HIE. The positive predictive value (PPV) of severe HIE was higher than that of an abnormal aEEG, and the PPV of moderate HIE was lower than that of an abnormal aEEG. The addition of aEEG pattern to HIE stage did not add to the predictive value. An abnormal aEEG was not independently associated with death or disability. (Shankaran S, Pappas A, McDonald SA, et al. Predictive value of an early amplitude integrated electroencephalogram and neurologic examination. Pediatrics July 2011;128(1):e112-e120). (Respond: Seetha Shankaran MD, Children's Hospital of Michigan, 3901 Beaubien St, 4 H46, Detroit, MI 48201. E-mail: sshankar@med.wayne.edu).

COMMENT. The Sarnat staging system (1976) provides a reliable clinical measure of severity of HIE in neonates that correlates well with neurodevelopmental impairment in infancy and childhood. An objective marker of the severity of HIE would assist in the selection of HIE cases eligible for clinical therapeutic whole-body hypothermia. The above study of the predictive value of an early, short duration aEEG in the outcome of HIE failed to demonstrate a positive effect of sufficient magnitude to be used alone as a marker. The associations are not as strong as those of previous studies of early aEEG and neurologic or developmental outcome. Weaknesses of the study cited by the authors include the brevity of the recordings, central electrode placement only, absence of serial and sleep-wake cycles of aEEG, and effects of seizures and anticonvulsants on the EEG background. 